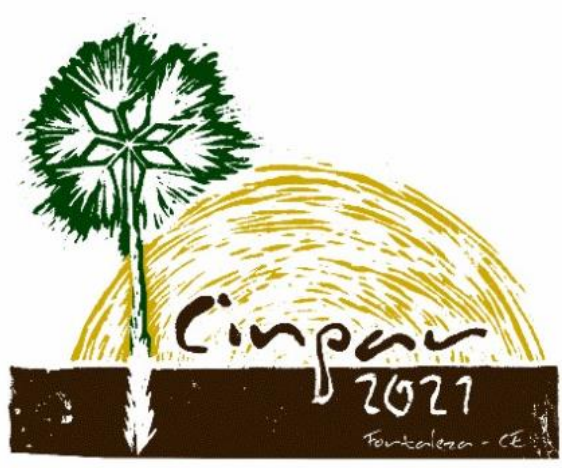

XVII Congresso Internacional sobre Patologia e Reabilitação das Construções

XVII Congreso Internacional sobre Patología y Rehabilitación de las Construcciones

XVII International Conference on Pathology and Constructions Rehabilitation

FORTALEZA (Brasil), 3 a 5 de junho de 2021

https://doi.org/10.4322/CINPAR.2021.002

\title{
Alteraciones y patologías en el patrimonio construido con tierra. La introducción del cemento en casos de estudio en el norte de Argentina
}

\author{
Jorge TOMASI ${ }^{1}$, JULIETA BARADA ${ }^{2}$ \\ ${ }^{1}$ CONICET / Laboratorio de Arquitecturas Andinas y Construcción con Tierra, Universidad Nacional de Jujuy, Tilcara, \\ Argentina, ju.barada@gmail.com \\ ${ }^{2}$ CONICET / Laboratorio de Arquitecturas Andinas y Construcción con Tierra, Universidad Nacional de Jujuy, Tilcara, \\ Argentina, jorgetomasi@hotmail.com
}

Resumen: La conservación y restauración del patrimonio construido con tierra ha estado sujeto a profundas revisiones técnicas y conceptuales en las últimas décadas en pos del establecimiento de consensos en torno al uso de materiales y procedimientos coherentes con las materialidades existentes. De todas maneras, persisten intervenciones en las que además de afectarse la autenticidad se generan diversos daños y degradaciones. Particularmente en el caso de Argentina, entre las décadas de 1940 y 1970 numerosos patrimonios construidos con tierra fueron intervenidos utilizando refuerzos de hormigón armado en diversos sectores de su mampostería de adobe con el objetivo mejorar su comportamiento estructural. Estas prácticas estaban vinculadas con el desconocimiento, pero también con los prejuicios asociados con estas técnicas constructivas. Si bien este tipo de intervenciones no persisten masivamente en la actualidad, su impacto en las estructuras es una problemática a abordar en los proyectos de conservación. Por su parte, el impacto que este tipo de prácticas ha tenido sobre las arquitecturas patrimoniales se ha extendido en la actualidad, al uso del cemento sobre otras construcciones de adobe.

En esta presentación se analizarán las patologías surgidas dese el uso de cemento para la restauración de la Iglesia de Uquía, en la Quebrada de Humahuaca, provincia de Jujuy, Argentina. El material a presentar surge del diagnóstico y proyecto realizados en los últimos años. Este trabajo ha mostrado los distintos deterioros producto de estas intervenciones, incluyendo sus consecuencias estructurales. De esta manera, se podrán analizar las implicancias que han tenido en el tiempo estas alteraciones. Al mismo tiempo, se analizará cómo estas intervenciones con cemento y hormigón armado en el patrimonio, se han multiplicado en otras construcciones de adobe contemporáneas y sus implicancias en las prácticas constructivas actuales y la sustentabilidad del patrimonio en la región.

Palabras clave: Área Andina, Mampostería de adobe, Hormigón armado

\section{Introducción}

Durante las primeras décadas del siglo XX en la Argentina comenzó la formalización de las acciones de protección de determinadas arquitecturas históricas desde las políticas estatales. Estas arquitecturas se asociarían con determinados valores relevantes en términos de la consolidación del ideario de un cierto proyecto nacional, en lo que sería el inicio del proceso de patrimonialización de numerosos bienes históricos. En este marco, también se comenzaron a desarrollar iniciativas más o menos sistemáticas, desde el mismo estado, para su conservación y restauración. En el marco de la primeras declaratorias como Monumento Histórico Nacional (MHN), las arquitecturas construidas con tierra, particularmente con adobe, tendrían una alta relevancia, con numerosos edificios reconocidos en torno a la década de 1940 (Herr, Rolón 2018). Esto 
estuvo vinculado con la importancia que esta técnica tuvo en momentos coloniales en buena parte del actual territorio argentino.

Mientras se reconocían los valores de algunas de estas construcciones a través de su declaratoria como MHN, el adobe era estigmatizado en torno a su asociación con la pobreza, el atraso, la falta de higiene o la precariedad estructural, en un proceso que comenzó a mediados del siglo XIX. Esta aparente contradicción se comprende al observar que el reconocimiento de los MHN se basaba en una valoración histórica, en relación con su antigüedad o los sucesos ocurridos en estos edificios, y no necesariamente por sus características técnicas específicas. El uso del adobe, entonces, no se constituía como un valor en sí mismo para justificar su declaratoria. En este contexto, de la mano con los prejuicios, existía un importante desconocimiento respecto a las mejores formas de intervenir en construcciones de adobe, que llevaron a la introducción de resoluciones técnicas incoherentes con los sistemas constructivos existentes y la incorporación de otras materialidades. El uso extensivo del cemento fue uno de los rasgos de estas intervenciones, considerando tanto su empleo en revoques, techos y también en estructuras de refuerzo de hormigón armado de distintas características. Si bien su utilización comenzó a mediados del siglo XX, continuó en las décadas subsiguientes e incluso en la actualidad. Pese a que el uso del cemento en construcciones de adobe se ha extendido en numerosos países y que se ha indicado la gravedad de las patologías asociadas (Rainer 2008), la interacción entre estas tecnologías no ha sido suficientemente estudiado.

El objetivo de esta presentación es precisamente describir los distintos modos en que se ha utilizado el cemento para la restauración de construcciones históricas en adobe, y analizar las patologías y daños asociados con su empleo, considerando las complejas interacciones emergentes entre estas materialidades y sus posibles remediaciones. El análisis se realizará a partir del trabajo de consolidación estructural y restauración integral realizado en la Iglesia de la Santa Cruz y San Francisco de Paula de Uquía, en la provincia de Jujuy, en el noroeste de Argentina. Este templo fue construido a finales del siglo XVII, declarado como Monumento Histórico Nacional en 1941, y fue objeto de una serie de acciones de restauración desde mediados del siglo XX que implicaron diferentes incorporaciones de cemento en distintas partes del edificio. Si bien el análisis se centrará en la Iglesia de Uquía, también se considerarán otros casos, con trabajos en distintos grados de avance, como la Casa del Marques en la localidad de Yavi y la Iglesia de Tabladitas, ambos edificios en las tierras altas también de la provincia de Jujuy (Figura 1).

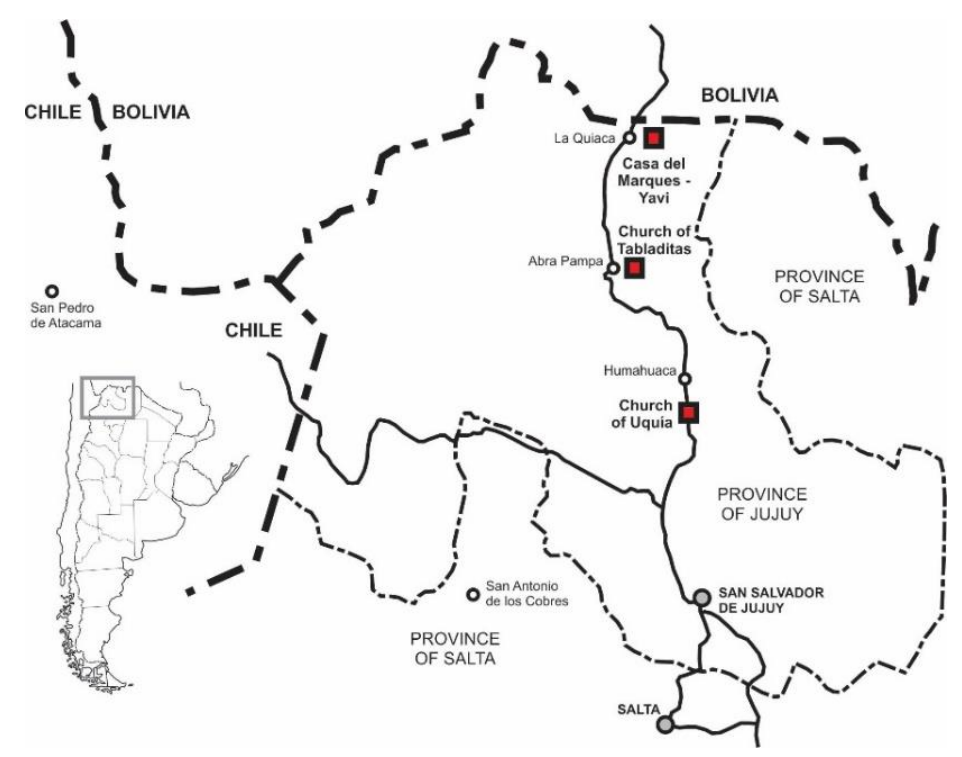

Figura 1 - Ubicación de los casos de estudio mencionados en el texto

El trabajo realizado en todos los casos implicó el registro y relevamiento de las construcciones, el diagnóstico de las patologías, el desarrollo de los proyectos y, en algunos de los casos, el seguimiento de las obras en terreno, a través de la asociación con organismos públicos y comunidades locales. En términos metodológicos, los proyectos se basaron en la articulación entre el trabajo de campo, estudio de 
documentación en archivo, análisis de materiales en laboratorio, evaluaciones estructurales a través del Método de Elementos Finitos (MEF) y el trabajo con técnicas participativas con las comunidades locales (Tomasi, Barada 2020).

\section{La conservación del patrimonio construido con tierra en la Argentina del siglo XX}

Si bien el registro de los edificios históricos, desde ámbitos académicos y gubernamentales, comenzó algunas décadas antes, la creación en 1938 de la Comisión Nacional de Museos y Lugares Históricos (Actualmente Comisión Nacional de Monumentos, de Lugares y de Bienes Históricos - CNMLyBH) implicó la formalización e integración de una política orientada a la protección y conservación de estas arquitecturas en todo el país. Dentro de las primeras declaratorias, entre 1938 y 1946, un total 122 MHN, el 45\% correspondía a arquitecturas construidas con tierra, mayormente adobe (Herr, Rolón 2018). Estas cifras dan cuenta de la relevancia que estás tecnologías tenían dentro del universo de los edificios valorados en las políticas de patrimonialización, correspondiendo en su mayoría a construcciones correspondientes a momentos coloniales emplazadas en el noroeste de Argentina y en la región de Cuyo, hacia el oeste. Tal como se indicó en la introducción, estas valoraciones estaban atravesadas por un criterio histórico, por lo que la tecnología constructiva no se presentaba como un elemento particularmente relevante. El foco puesto en las construcciones coloniales, como la propia Iglesia de Uquía, estaba estrechamente vinculado con las reivindicaciones nacionalistas de principios del siglo XX (Tomasi 2012). Las primeras intervenciones sobre estos MHN, de hecho, más que guiarse por criterios de autenticidad, se orientaron a la creación de imágenes idealizadas de las arquitecturas coloniales en el país, con transformaciones relevantes en su materialidad (Figura 2).

En el caso de los MHN construidos con tierra, los prejuicios asociados con la capacidad estructural de estas técnicas y su durabilidad, sumado al desconocimiento general sobre los mejores criterios a aplicar para su conservación, llevaron a la introducción de otras materialidades para la consolidación estructural de los edificios, como el cemento y las estructuras metálicas. El uso del cemento, particularmente, se extendió tanto para el reemplazo de los revoques de barro, los cambios en los techos de tierra y la introducción de elementos estructurales de hormigón armado. Más allá de las particularidades del uso de este material en los distintos elementos constructivos, en todos los casos existe un factor común asociado a la idea de que tanto el barro como el adobe en sí se constituían como materiales de escasa durabilidad y de extrema debilidad frente al agua. Esta idea, sumada al desconocimiento sobre las técnicas convergió en el uso del cemento como material de reemplazo, considerando su supuesta durabilidad e impermeabilidad como factores sustanciales.
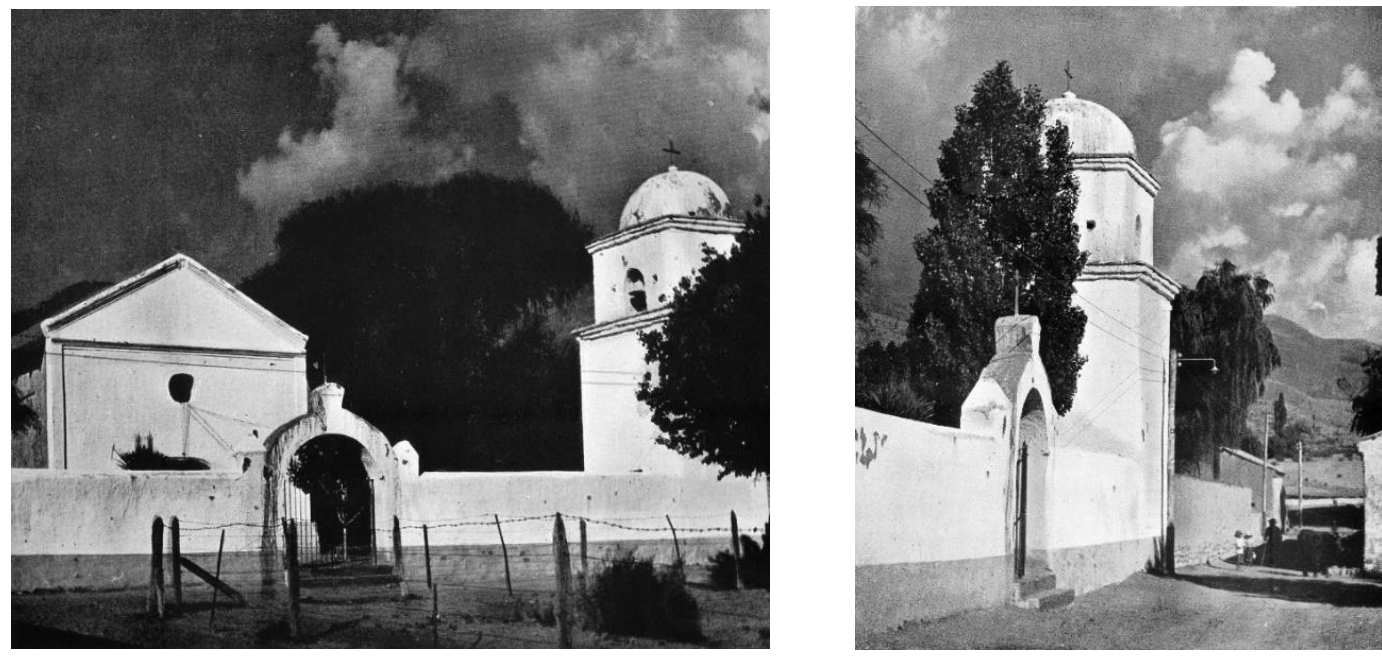

Figura 2 - La Iglesia de Uquía en 1939, previo a su declaratoria como MHN (ANBA 1939)

El sismo de 1944 en la ciudad de San Juan (7.4 en la escala de Richter), en la región de Cuyo al oeste del país, provocó una destrucción generalizada, afectando particularmente a las construcciones de adobe que no 
contaban con elementos de refuerzo. Este evento contribuyó al incremento de los prejuicios y el rechazo al uso del adobe, tanto por razones estructurales como sanitarias, y al mismo tiempo el aliento al uso de estructuras de hormigón armado como única solución viable para la construcción en zonas sísmicas (Healey 2009). Entre las décadas de 1940 y 1950, incluso, se extendió el uso del hormigón armado para el refuerzo de numerosas construcciones de adobe (Viñuales 2011). En este marco, durante esos años, un porcentaje relevante de los MHN construidos con adobe tuvieron intervenciones generalizadas que implicaron alteraciones en sus sistemas constructivos, afectando su autenticidad, pero a la vez generando diversas problemáticas técnicas, siendo la Iglesia de Uquía uno de los casos más relevantes.

Si bien la incorporación de cemento en construcciones con tierra no ha sido una temática particularmente trabajada, sí se han documentado casos en distintos países involucrando edificios de adobe o de cob, como Brasil, Inglaterra o México (Pecoraro 1993; Keefe et al. 2000; Guerrero Baca 2019), donde se observaron problemáticas similares a las referidas en este trabajo. En el caso de Nuevo México, Estados Unidos, se ha referido que el cemento comenzó a incorporarse como material para los revoques en la década de 1930 (Cornerstone 2006), coincidente con los procesos referidos en el caso de Argentina.

\section{La Iglesia de la Santa Cruz y San Francisco de Paula. Materialidades e intervenciones}

La Iglesia de la Santa Cruz y San Francisco de Paula se construyó en torno a 1691 (Viscontini 1968), en el poblado de Uquía, en el marco de los procesos de consolidación del control colonial y las políticas de evangelización de la Quebrada de Humahuaca (Sica 2014), en el noroeste del actual territorio argentino, dentro de una red de poblados destinados a la reducción de la población indígena. La capilla, que se constituía como el eje del poblado, responde en términos arquitectónicos-constructivos a las características generales de los templos de la región, aunque con una serie de particularidades técnicas. Se conforma a partir de una nave única de 17,5 $\mathrm{m} \times 7,15 \mathrm{~m}$, orientada hacia el este, con una cubierta a dos aguas y una altura interior de $8 \mathrm{~m}$ hasta la cumbrera. En el lado norte tiene una sacristía adosada de $6 \mathrm{~m}$ de lado (Figura 3). A diferencia de otros casos cuyas cubiertas conforman capillas abiertas en el frente, la Iglesia de Uquía tiene una fachada telón, plana, con un remate triangular que cubre la estructura del techo. El muro perimetral delimita un atrio de $24 \mathrm{~m}$ de frente por $34 \mathrm{~m}$ de profundidad, con la única torre exenta de la nave, ubicada en la esquina noreste. La torre tiene una base de $4 \mathrm{~m}$ de lado, y una altura de $9,9 \mathrm{~m}$ (Figuras 4 y 5 ).
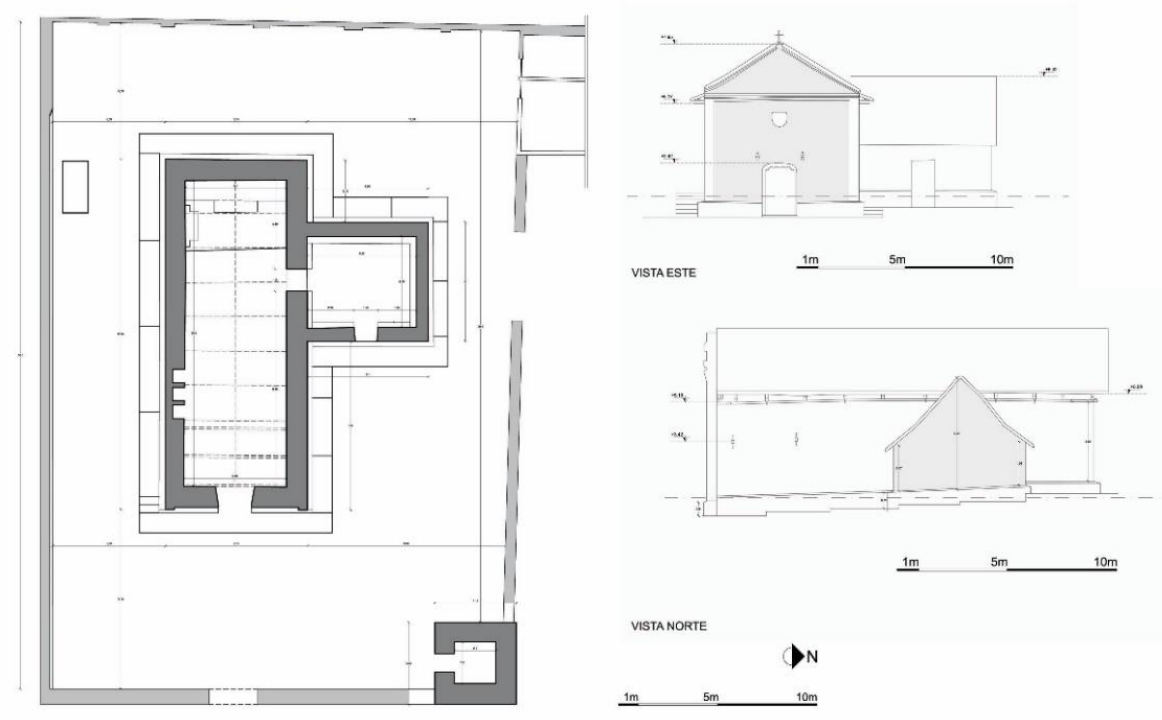

Figura 3 - Planta y vistas de la Iglesia de Uquía

En términos constructivos, la capilla presenta muros de adobe, de $60 \times 30 \times 10 \mathrm{~cm}$, en muros de $1 \mathrm{~m}$ de espesor en la nave, y de $65 \mathrm{~cm}$ en la sacristía. El sobrecimiento alcanza los $80 \mathrm{~cm}$ de altura y es de piedras medianas a grandes asentadas con mortero de barro con cal agregada. La Iglesia de Uquía presenta 
elementos significativos en la estructura de madera del techo, conformada por cerchas de par y nudillo. En el coronamiento de los muros presenta una doble viga continua de madera, sobre la que se apoyan las cerchas, que recorre todo el perímetro tomando la totalidad del espesor, con las dos piezas a filo del muro. Este sistema de viga collar no solo contribuye a la estabilidad general, sino que además mejora el apoyo de la estructura de la cubierta y su vinculación con el muro. Sobre la viga collar se apoyan los canes que soportan 8 cerchas con tensor inferior, y sobre éstas se ubican las alfajías que a su vez soportan el entablonado de madera de cardón, sobre el que se ejecutó la terminación con una torta de barro de alrededor de $10 \mathrm{~cm}$ de espesor. La presencia de la viga collar es una cuestión relevante en relación con la solidez estructural general del edificio, particularmente considerando que se encuentra en una zona de riesgo sísmico 2 (moderada).


Figura 4 - Vista del frente de la capilla, con su torre exenta, antes de la intervención actual

Figura 5 - Vista posterior, con el encuentro de la nave y la sacristía, antes de la intervención actual

Si bien desde comienzos del siglo XX la Iglesia de Uquía fue estudiada por su valor histórico, desde el campo académico en el marco de reivindicaciones del pasado colonial (Tomasi 2012), su declaratoria como Monumento Histórico Nacional en 1941 implicó la formalización de su proceso de patrimonialización. Si hasta ese momento la conservación del edificio había sido responsabilidad de la comunidad local, a partir de ese momento se iniciaron las intervenciones basadas en la acción de los organismos públicos. La primera de estas intervenciones, y probablemente la más importante, se realizó durante la década de 1940. Dada la falta de documentación, los trabajos realizados debieron reconocerse a partir de la comparación de fotografías. Esta restauración es muy relevante para los objetivos de este texto puesto que implicó la incorporación de los refuerzos de hormigón armado que se analizarán, junto con cambios morfológicos en la torre, entre otras alteraciones (comparar las figuras 2 y 4 para observar la modificación de la torre). Si bien existen referencias a otras intervenciones en la década de 1960 (Viscontini 1968), la siguiente restauración documentada se llevó adelante a finales de la década de 1980, con modificaciones en las características del techo de la nave, que implicaron la incorporación de materialidades ajenas a la técnica de la torta de barro. Entre el 2012 y el 2018 se volvieron a realizar trabajos sobre el techo de la iglesia, con nuevos cambios en la técnica ejecutada, que tampoco lograron mejorar su impermeabilidad y durabillidad.

\section{Las presencias del cemento y sus patologías}

Dados los daños generales que se registraban en la iglesia, en el 2019 se comenzó a realizar un trabajo de registro y diagnóstico del estado que presentaba el edificio para la elaboración de un proyecto de intervención para su consolidación y restauración, que finalizó en el 2021. El diagnóstico mostró una serie de daños estructurales en los muros de adobe, con cuatro grietas verticales escondidas detrás de los revoques, y problemas de humedad generales producto de las filtraciones en el techo (Barada, Tomasi 2020; Tomasi, Barada 2020). El estudio del mecanismo de los daños mostró que, si bien los problemas estructurales estaban asociados con defectos constructivos propios del templo, las intervenciones con materiales ajenos a la materialidad existente no solo no dieron resultados adecuados, sino que provocaron nuevas patologías. El 
uso de cemento fue uno de los rasgos principales de las intervenciones desde mediados del siglo XX y comienzos del XXI, mostrando la continuidad hasta el presente de este tipo de resoluciones. Esta presencia del cemento puede clasificarse en torno a su uso en elementos estructurales, como los refuerzos de hormigón armado en los muros de adobe, la aplicación de revoques de cemento en reemplazo de los originales de tierra, y la aplicación de morteros de cemento en la torta de barro del techo con el objetivo de aumentar su durabilidad.

\subsection{Elementos estructurales}

Si bien el estudio de la documentación contenida en la CNMLyBH mostró la existencia de intervenciones previas sobre el edificio, no existían referencias a la introducción de elementos de hormigón armado, más allá del cambio del remate de la torre. Los cateos preliminares mostraron la existencia de elementos de hormigón armado en los muros, aunque al retirar los revoques se pudo registrar la incorporación de una estructura integral conformada por columnas y vigas (encadenado superior e inferior) que recorre la nave de la iglesia y la sacristía, y que se encuentran empotradas en los muros desde el exterior (Figuras 6 y 7 ).
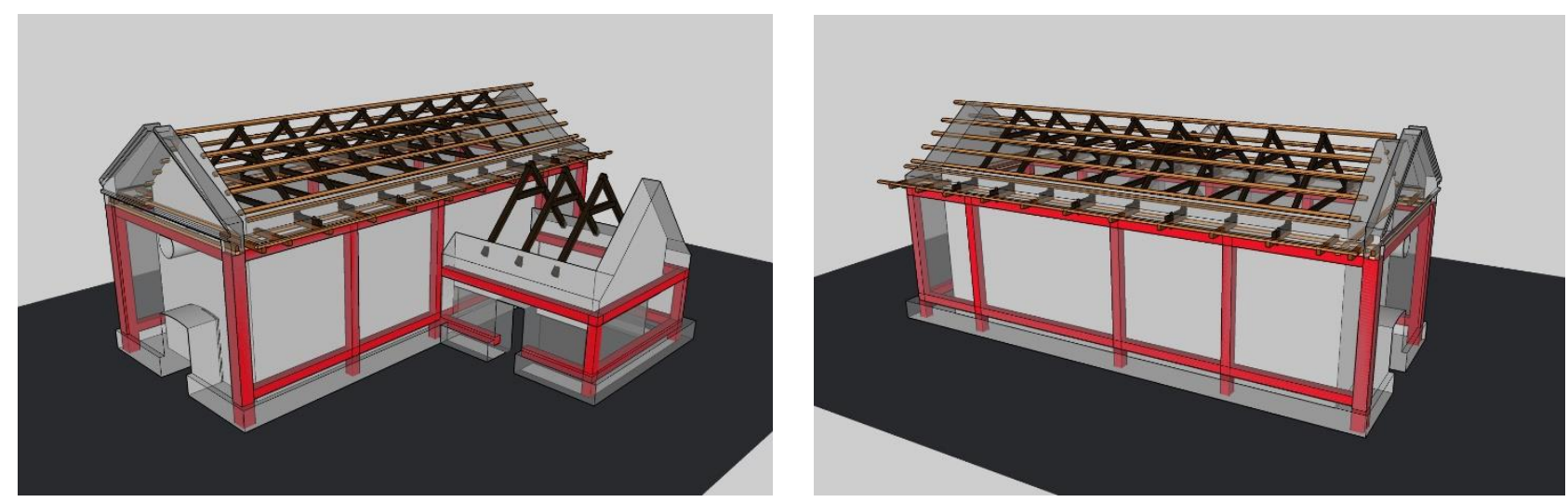

Figure 6 - Esquema de la Iglesia con la estructura de hormigón armada indicada en rojo
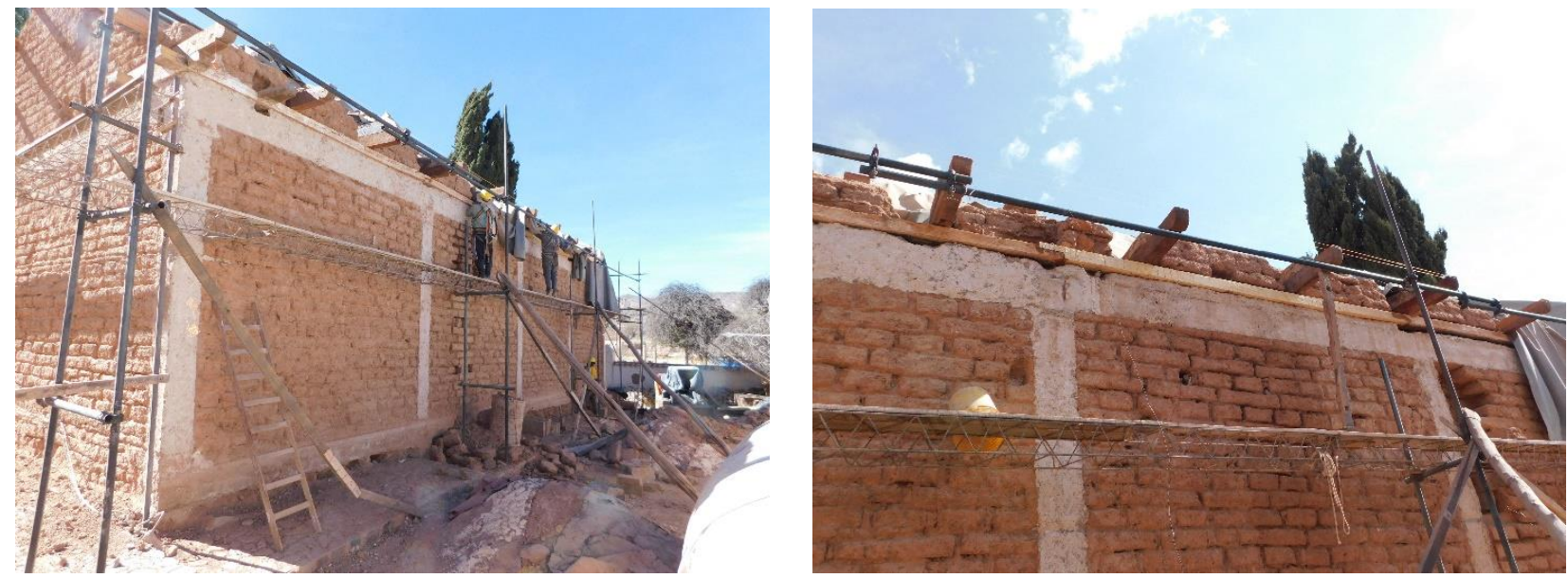

Figura 7 - Vista y detalle de la estructura de hormigón armado en los muros laterales

La viga de encadenado superior, de $30 \mathrm{~cm} \times 30 \mathrm{~cm}$, se encuentra ubicada debajo de la viga collar de madera, mientras que la inferior se encuentra a $0,71 \mathrm{~m}$ desde el nivel del suelo, por encima del sobrecimiento de piedra, estando ausente en el muro frontal. Las 10 columnas se ubican a una distancia aproximada de $4 \mathrm{~m}$ en los muros laterales, a las que se suman dos en las esquinas de la sacristía. También tienen una sección de 30 $\mathrm{cm} \times 30 \mathrm{~cm}$, a excepción de las dos localizadas en el frente, donde sobresalen como pilastras, con una sección de $40 \mathrm{~cm} \times 40 \mathrm{~cm}$. En todos los casos las columnas alcanzan una profundidad de 1,30 m, sin otra fundación observable. Las vigas y columnas presentan una armadura con 4 hierros de $10 \mathrm{~mm}$, lisos, que en distintos sectores por el deterioro en el hormigón presentan un estado de oxidación avanzado. 
Como se mencionó, el diagnóstico realizado permitió detectar cuatro grietas pasantes verticales en los muros laterales, probablemente surgidas por el asentamiento del frente de la nave, y que podrían corresponder a las grietas referidas en un documento de 1912. En ese caso, esta estructura de hormigón armado, probablemente, fue incorporada a los efectos de consolidar estructuralmente un edificio que presentaba ciertos daños, aunque con una intervención sumamente invasiva, que no habría dado respuesta al problema de base, que ya se encontraba estabilizado. Debe considerarse que se trata de elementos estructurales de una sección reducida $(30 \mathrm{~cm} \times 30 \mathrm{~cm}$ ) insertos en uno de los lados de muros de hasta $1 \mathrm{~m}$ de espesor, por lo que su aporte en la consolidación del edificio es muy limitado. De hecho, el modelado a través del MEF mostró que la estructura de hormigón armado y la mampostería de adobe en la actualidad tienen un comportamiento independiente y autoportante, aunque la primera podría amplificar las debilidades del muro de adobe producto de las rigideces disímiles, particularmente frente a un evento sísmico.

La Casa del Marqués, también una construcción colonial del siglo XVII ubicado en la localidad de Yavi, y MHN desde el 2001, también fue objeto de intervenciones que implicaron la incorporación de elementos estructurales de hormigón armado, aunque en este caso se trató de una viga de encadenado superior de una sección de $50 \mathrm{~cm} \times 25 \mathrm{~cm}$ sobre muros de adobe de $1 \mathrm{~m}$ de espesor. Dados los daños existentes, en este caso se pudo observar cómo el apoyo de esta viga generó efectos de corte en el interior del muro, con grietas internas que podían provocar colapsos parciales en el muro (Barada, Tomasi 2020). Las intervenciones realizadas implicaron la toma de decisiones respecto de la permanencia de estos elementos, que no implican un criterio uniforme. En el caso de Yavi, al tratarse de un elemento colocado sobre el muro, el hormigón armado pudo reemplazarse por una viga continua de madera, mientras que la Iglesia de Uquía, al tratarse de una estructura integral, cualquier procedimiento de retiro hubiera implicado mayores daños, por lo que debió consolidarse la mampostería de adobe.

Más allá de estas incorporaciones estructurales generales, tanto la Casa del Marqués como la Iglesia de Uquía presentan, además, elementos de hormigón armado puntuales, colocados a modo de refuerzo, en ciertos sectores de la mampostería de adobe. En el caso de la Iglesia se registró un dintel aislado, con una sección de $30 \mathrm{~cm} \times 30 \mathrm{~cm}$, sobre los confesionarios, sin vinculación alguna con el resto del muro (Figura 8). Producto de las diferencias en la rigidez entre los elementos y sus frecuencias de vibración, sus interacciones durante un sismo pueden provocar el colapso del muro de adobe, tal como surge de investigaciones desarrolladas en Perú (MVCyS 2014). También en la Iglesia, se debe considerar el cambio del remate de la torre. La cúpula de adobe que se observa en las primeras fotografías, fue reemplazada por una pieza piramidal de hormigón armado que está simplemente apoyada sobre los muros de adobe (Figura 9). Para el análisis de este elemento también se realizó un modelado que mostró que, si bien este remate y el muro de adobe tendrán un comportamiento independiente frente a los esfuerzos en un sismo, su retiro podía generar daños mayores.


Figura 8 - Dintel de hormigón armado sobre los confesionarios en la nave de la iglesia

Figura 9 - Vista del interior del remate piramidal sobre la torre 


\subsection{Revoques}

El reemplazo de los revoques de barro por otros de cemento en construcciones de adobe, históricas y contemporáneas, es una práctica que se ha masificado en distintos lugares del mundo. Esto ha estado también vinculado con los prejuicios asociados con la tierra como material, pero específicamente con una búsqueda de aumentar la durabilidad de los revoques frente a la incidencia de los agentes ambientales, particularmente el agua. Sin embargo, distintas investigaciones han mostrado cómo el uso del cemento es el origen de distintas patologías que pueden incluir el desarrollo de daños estructurales graves en las construcciones de adobe (Cornerstone 2006; Rainer 2008; Tomasi, Barada 2020). Una de las propiedades de las diversas técnicas de construcción con tierra, incluyendo al adobe, es su capacidad para intercambiar vapor con el ambiente. Esto tiene una incidencia importante en relación con sus propiedades térmicas, pero también permite la evaporación del agua que pudo haber ingresado en los muros producto de la capilaridad, la incidencia directa de las lluvias o las filtraciones en los techos. Al cubrir los muros con revoques de cemento, la humedad tiende a encapsularse en el interior, al no poder evaporarse, sosteniendo su acción en el tiempo. Producto de esto, la humedad por capilaridad podrá alcanzar una altura mayor en el muro, dada la imposibilidad de evaporarse. La acción sostenida de la humedad puede llevar a la disgregación del material en los adobes, reduciendo su resistencia en forma significativa, particularmente en la base de los muros. En este sentido, la aplicación de cemento no reduce la acción de la humedad, sino que la amplifica, ocultando sus efectos (Cornerstone 2006).

A lo largo de las distintas intervenciones en el tiempo que tuvo la Iglesia de Uquía, los revoques de barro se fueron reemplazando con otros de cemento, en especial en las caras exteriores de los muros y en los tramos inferiores de los interiores. Estas aplicaciones se dieron de distintas maneras en cada intervención, con diferentes espesores y combinaciones con los morteros de barro. En este sentido, durante el diagnóstico se registraron desde revoques de cemento superficiales, de menos de $5 \mathrm{~mm}$, sobre los de barro, hasta aplicaciones de cemento de hasta $5 \mathrm{~cm}$ de espesor directamente sobre el muro de adobe (Figuras 10 y 11). Los revoques de cemento mostraban una cantidad importante de fisuras como consecuencia de la diferencia de rigideces entre este material y el soporte de adobe, y de la limitada capacidad del cemento para absorber la contracción y dilatación provocada por la gran amplitud térmica de la región.


Figura 10 - Detalle de un revoque de cemento sobre el muro de adobe

Figura 11 - Detalle de una capa superficial de cemento sobre el revoque de barro

Una de las principales patologías detectadas en el edificio estuvo vinculada con la presencia de humedad tanto por capilaridad como por fallas en la cubierta, que se analizarán en el próximo punto, que generaron filtraciones en el tramo superior de los muros. La aplicación de revoques cementicios en los muros provocó que la humedad se acumulara en el tiempo, sin poder evaporarse hacia el ambiente. En los cateos realizados 
en los sectores afectados en los muros mostraron que, pasados 7 días de la incidencia de precipitaciones, los adobes de las hiladas afectadas por la humedad sostenían un $12 \%$ de contenido de agua, medida por peso, frente al $2 \%$ esperable en condiciones normales. Las principales patologías asociadas con esta incidencia de la humedad se concentraban en las 5 a 10 hiladas superiores de adobe $(50$ a $100 \mathrm{~cm})$, dependiendo del sector del muro, y en todos los casos involucrando a la viga collar y los apoyos de la estructura de madera (Figura 12). La imposibilidad de evaporación de la humedad hacia el exterior, por el uso de los revoques de cemento, favoreció la extensión de su incidencia en el muro, abarcando más hiladas de la mampostería de adobe.
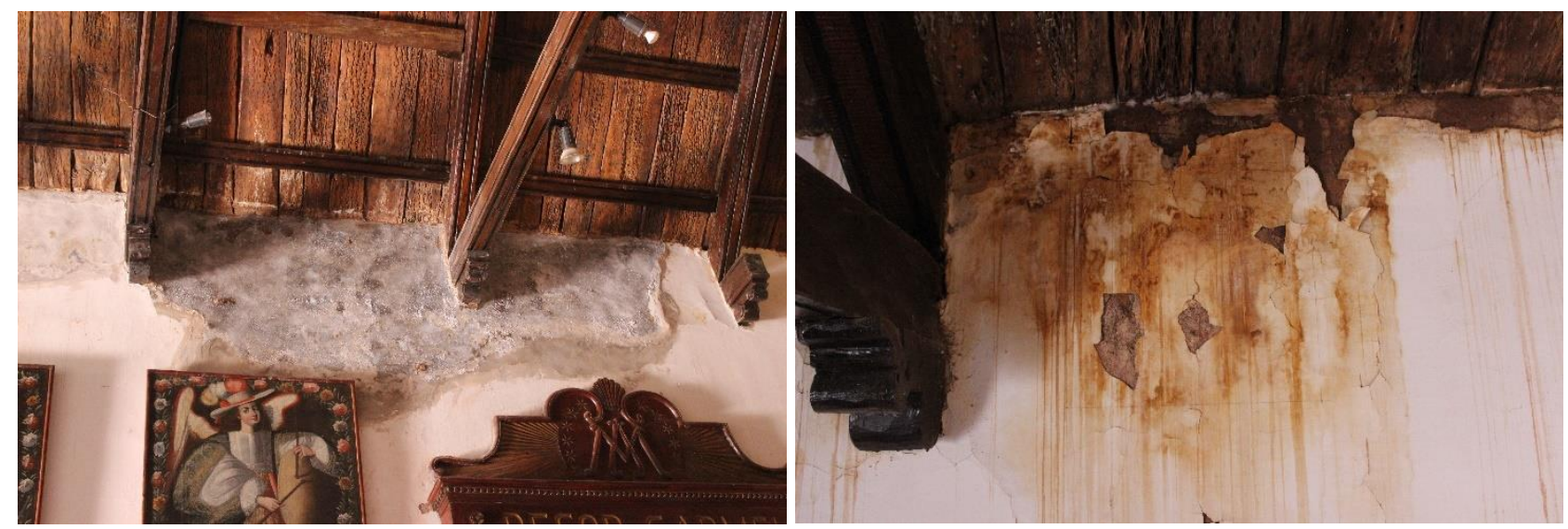

Figura 12 - Daños provocados por la humedad en el tramo superior de los muros

Si bien en algunos de los sectores la humedad tenía una expresión visible, los revoques de cemento ocultaron el verdadero alcance de los daños existentes. Esta exposición de los materiales a una humedad sostenida en el tiempo tuvo implicancias importantes en relación con el disgregamiento de los adobes y su pérdida de cohesión y por ende de resistencia, la formación de hongos, el biodeterioro de los materiales orgánicos como las vigas de madera del techo y, particularmente el cielorraso de cardón. Esto incluso generó la fractura de los tensores en el apoyo, producto de la pérdida de resistencia como consecuencia de la degradación del material. Problemáticas similares se observaron tanto en la Casa del Marques como en la Iglesia de Tabladitas, donde el deterioro de la estructura del techo contribuyó a la pérdida de estabilidad de los muros.

\subsection{Cubiertas}

Los techos de tierra han sido de una de las materialidades más afectadas por la introducción de materiales ajenos al sistema constructivo, incluyendo a los techos de torta de barro como el de la Iglesia de Uquía. Esta técnica consiste en la ejecución de una o dos capas continuas de tierra en estado plástico, con distintos agregados para su estabilización, particularmente fibras vegetales (Tomasi 2013). Si esta técnica se ejecuta correctamente brinda una adecuada aislación hidrófuga y térmica, al tiempo que permite la evaporación de la humedad en el interior de los ambientes. Además del escurrimiento del agua producto de la pendiente del techo, la torta de barro absorbe el agua durante las lluvias y luego la libera al ambiente. Por sus características, la torta de barro requiere de un mantenimiento periódico con el reemplazo parcial o total del material que se erosiona a lo largo del tiempo. Esta operación debe repetirse cada 2 a 5 años, dependiendo de las condiciones ambientales, del tipo de suelo utilizado y de su correcta ejecución. Producto de esta necesidad de mantenimiento, en las últimas décadas se han intentado distintas modificaciones en esta técnica, introduciendo distintos materiales como capas de mortero de cemento, inferiores o superiores, o membrana líquida. En todos los casos estos materiales bloquean o limitan la capacidad de intercambio de vapor con el ambiente, con una lógica similar a la indicada para el caso de los revoques. Si bien los morteros de cemento impiden la evaporación de la humedad, no impiden las filtraciones. Por la rigidez del material, al no poder absorber las dilataciones y contracciones asociadas con la amplitud térmica diaria, las capas de cemento tienden a fisurarse, lo que permite el ingreso de agua en determinados sectores. 
En el caso de la Iglesia de Uquía, las principales transformaciones en la materialidad del techo se dieron entre el 2012 y el 2018, lo que muestra la persistencia en la aplicación de criterios erróneos para la conservación del patrimonio construido con tierra. En lugar de las capas continuas de barro, estas intervenciones implicaron complejas interacciones entre distintos materiales. En primer lugar, se ejecutó una capa de mortero de cemento de $2 \mathrm{~cm}$ de espesor sobre una base de cartón. Sobre esta capa se dispuso alambre romboidal -que al día de hoy presentaba un importante nivel de oxidación-, con listones de madera de pino, seguramente para evitar el deslizamiento de una torta de $8 \mathrm{~cm}$ de espesor que se realizó encima. Como capa de terminación se colocó una malla de fibras plásticas, clavada sobre los listones, que además se pintó con membrana líquida roja (Figura 13).
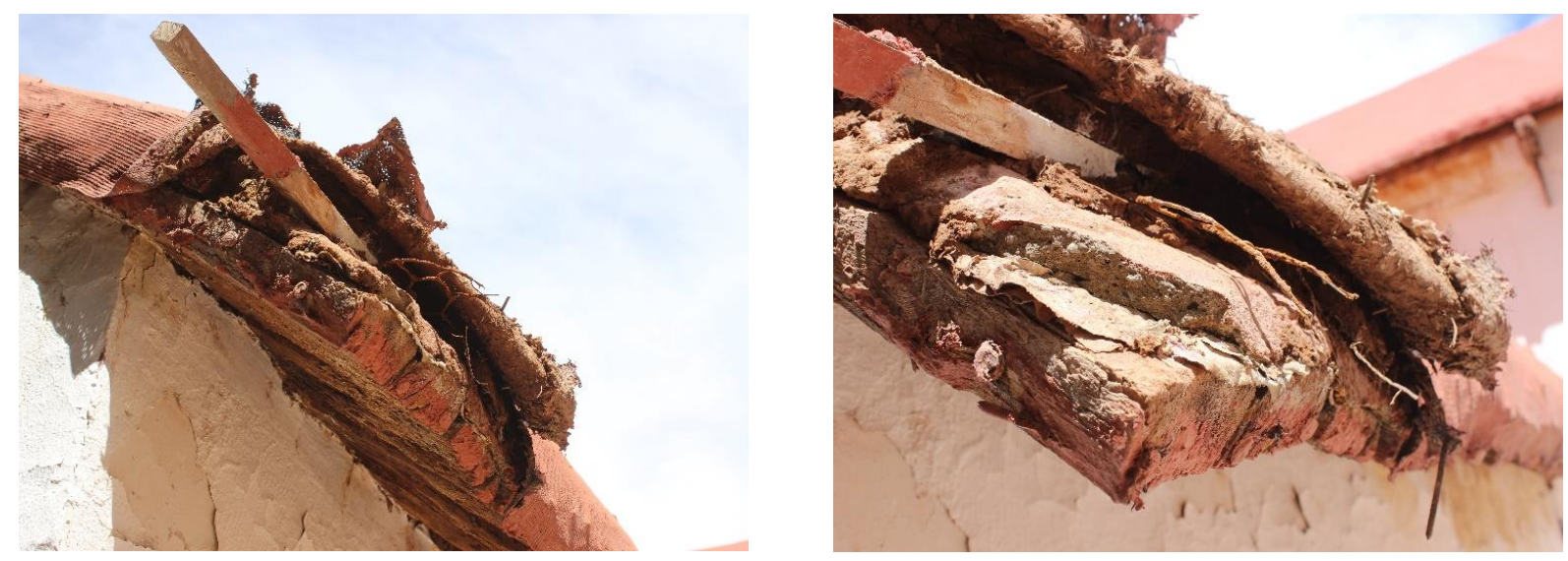

Figura 13 - Detalles de las transformaciones en la torta de barro del techo

Con el paso del tiempo, la membrana se degradó producto de la radiación solar permitiendo el ingreso de agua a la capa de barro que, al no poder evaporarse, se acumulaba en el material. De hecho, al momento de su estudio esta capa de barro mostraba un alto nivel de humedad, por encima del $10 \%$ en peso. Esto muestra que la resolución no solo no evitó el ingreso de agua, sino que además impidió la adecuada evaporación y secado del material, amplificando los deterioros. Por la exposición continua a la humedad, la capa de cemento de base, al no ser hidrófuga, permitió filtraciones hacia los muros que provocaron los daños que se indicaron en el punto anterior tanto en los elementos de madera como en la mampostería de adobe, donde tampoco pudo evaporarse por la presencia de los revoques de cemento.

\subsection{Transiciones}

La construcción con adobe no solo tiene una presencia relevante en las construcciones históricas en el área de estudio, sino que además tiene una incidencia muy importante en la arquitectura doméstica contemporánea. En la actualidad, el adobe es el material predominante en más del $80 \%$ de las viviendas, en buena parte de la provincia de Jujuy, mostrando la persistencia de esta técnica, pese a los referidos procesos de estigmatización. Más allá de esta persistencia, los modos de hacer y las formas de utilización de la mampostería de adobe, sin bien nunca fueron estáticos, en las últimas décadas han atravesado procesos de transformación intensos. Estos cambios han estado vinculados con una reducción del espesor de los muros, que hoy tienden a no superar los $20 \mathrm{~cm}$, con el consiguiente aumento de la esbeltez de las construcciones y la reducción de su capacidad portante y de su capacidad para soportar esfuerzos horizontales, junto con un aumento de las dimensiones de las aberturas, que también es nocivo para la resistencia estructural de la mampostería de adobe. De la mano con estos cambios, y en parte en relación con ellos, en las arquitecturas domésticas contemporáneas también se ha multiplicado el empleo del cemento en formas muy similares a las que se han referido para la restauración de las construcciones históricas, como la Iglesia de Uquía, o la Casa del Marques. Cabría preguntarse si el empleo de este material en construcciones emblemáticas de la región no ha contribuido a una ampliación de su uso en otras arquitecturas.

En relación con la incorporación de elementos estructurales, se ha masificado la ejecución de estructuras integrales de vigas y columnas en las obras nuevas, que luego tienen al adobe como material de cerramiento 
no portante. Incluso este tipo de estructuras son incorporadas en construcciones existentes, en el marco de reparaciones o ampliaciones de las viviendas (Figura 14). En cierto modo, el uso de estas estructuras de hormigón armado actúa como una justificación para la reducción de los espesores de los muros de adobe, aunque no se considera que ambos materiales tendrán comportamientos independientes, puesto que no habrá un vinculo material entre ambos. Por esta razón, el muro de adobe podrá colapsar o volcarse, por fuera del cuadro de la estructura. En otras construcciones, el hormigón armado, tal como se ha observado en el caso de Uquía o la Casa del Marques, se utiliza en refuerzos aislados dentro de las construcciones, como viga de encadenado superior o en la forma de elementos puntuales como dinteles sobre puertas y ventanas.
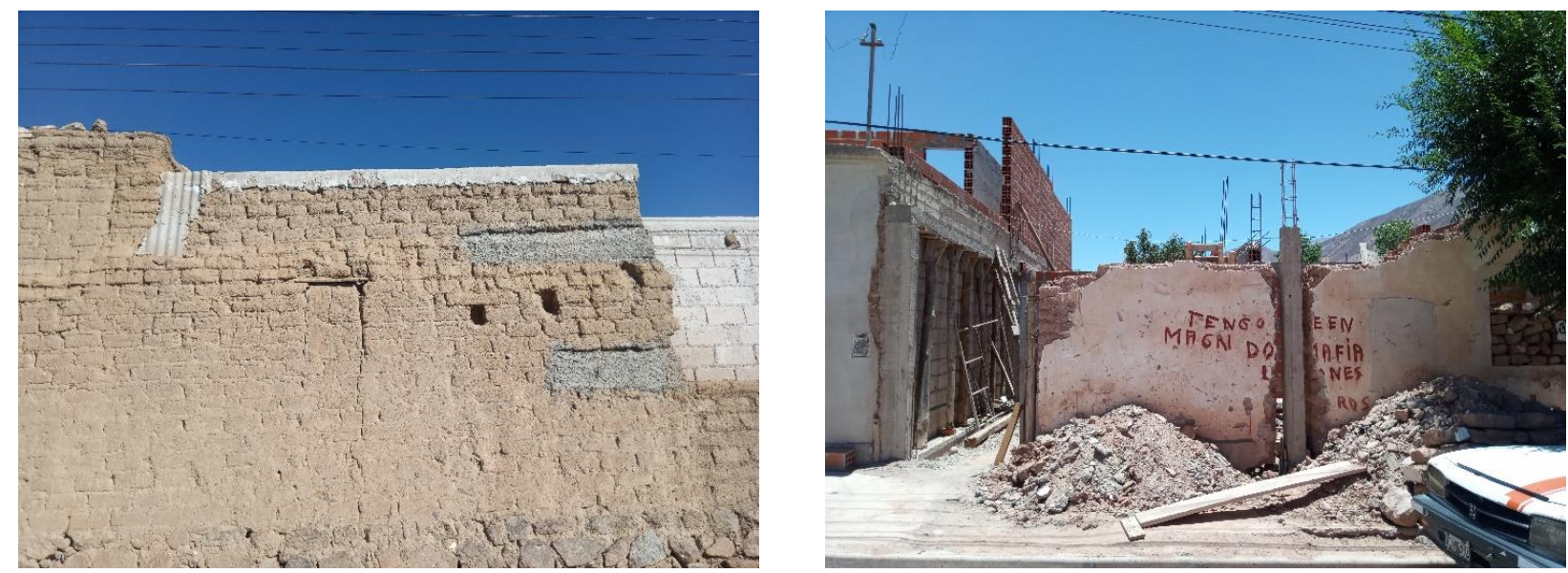

Figura 14 - Introducción de elementos de hormigón armado en muros de adobe

Los revoques de cemento también se han masificado al punto de ser hoy en día la situación más habitual en las construcciones de adobe de la región, pese a ser una causa potencial de daños graves en los muros de adobe, y lo propio ocurre con la introducción del cemento en las terminaciones de torta de barro. En ambos casos, la acumulación de humedad por la imposibilidad de su evaporación natural hacia el ambiente, pueden ser el origen de mecanismos de disgregación del material de los adobes, con potenciales consecuencias estructurales, tal como se ha analizado más arriba.

\section{Conclusiones}

El empleo del cemento en relación con lo construcción con tierra en general, y en la mampostería de adobe en particular, comenzó a utilizarse en la primera mitad del siglo XX en Argentina y en otros países, tanto en refuerzos estructurales a través del hormigón armado, como en el reemplazo de los revoques de barro. Estos usos emergieron de los prejuicios hacia la construcción con adobe y los discursos sobre la falta de higiene, el atraso y la debilidad estructural, como con la maximización de los beneficios asociados con el cemento. Más allá que se han discutido estos usos del cemento, desde la teoría de la conservación y desde los estudios sobre los materiales, muchas de estas prácticas se siguen observando en restauraciones recientes. Las primeras aplicaciones estuvieron vinculadas con la conservación de edificios históricos, en el marco de políticas públicas explícitas, sin embargo, en la actualidad se han masificado involucrando todo tipo de construcciones con adobe. Las interacciones entre el adobe y el cemento no solo no contribuyen a una mejora de las prestaciones, sino que pueden ser el origen de patologías graves que pueden poner en riesgo la estabilidad de los edificios y a las personas que los habitan.

El caso de la Iglesia de Uquía que se ha analizado en este trabajo se constituye como un ejemplo relevante para analizar estas problemáticas. Desde el momento de su declaración como MHN, esta capilla del siglo XVII estuvo sujeta a distintas intervenciones en las que se fueron aplicando distintas resoluciones incompatibles basadas en el uso del cemento, incluyendo refuerzos estructurales de hormigón armado, revoques y terminaciones en los techos. Las fallas en las resoluciones ejecutadas en la torta de barro favorecieron el desarrollo de filtraciones que afectaron gravemente a los muros de adobe y los elementos de madera de la 
estructura del techo. La aplicación de revoques de cemento provocó que no pudiera evaporarse la humedad, generada por estas filtraciones en los tramos superiores de los muros y por capilaridad en los inferiores, amplificando los daños. El sistema de vigas y columnas de hormigón armado incorporado a la mampostería de adobe tampoco contribuyó a mejorar el comportamiento estructural del edificio producto de su comportamiento independiente, mientras que sí puede constituirse como el origen de nuevos daños frente a un movimiento sísmico, además de las evidentes afectaciones a la autenticidad.

Como lo han mostrado distintas investigaciones, la mejora de las prestaciones y durabilidad de las construcciones con adobe, y el incremento de su capacidad resistente frente a los sismos es un desafío importante tanto para la conservación de las construcciones históricas como para producción contemporánea con esta técnica. Sin embargo, el verdadero desafío es lograr estas mejoras desde el conocimiento de las propiedades del material con soluciones compatibles y sustentables.

\section{Referencias}

Barada, J., Tomasi, J. (2020) (Dis)continuidades constructivas en la restauración del patrimonio en tierra del noroeste argentino. Loggia, Arquitectura \& Restauración, 33 (2020), 44-57.

Cornerstones Community (2006). Adobe Conservation. A Preservation Handbook. Cornerstones Community, Santa Fe.

Guerrero Baca, F. (2019). Comportamiento sísmico de viviendas tradicionales de adobe, situadas en las faldas del volcán Popocatépetl, México. Gremium, 6-11, 105-118.

Healey, M. (2009). The "Superstition of Adobe" and the Certainty of Concrete. Shelter and Power after the 1944 San Juan Earthquake in Argentina, in Buchenau, J., Johnson, L. (Eds). Aftershocks: Earthquakes and Popular Politics in Latin America. University of New Mexico Press, Albuquerque, 100-128.

Herr C., Rolón, G. (2018). Registro documental e intervención patrimonial en la arquitectura religiosa de la provincia de Jujuy. Criterios implementados por la Comisión Nacional de Museos, de Monumentos y Lugares Históricos (CNMMYLH) durante el período 1938-1946. Anales del IAA, 48 (1), 31-45.

Keefe, L., Watson, L., Griffiths, R. (2000). Possible causes of structural failure in traditional cob buildings, in Terra 2000: 8th International Conference on the Study and Conservation of Earthen Architecture. James and James, London, 254-260.

Ministerio de Vivienda, Construcción y Saneamiento (2014). Fichas para la Reparación de Viviendas de Adobe. Ministerio de Vivienda, Construcción y Saneamiento, Lima.

Pecoraro, A.L. (1993). The conservation of the Church of Nossa Senhora do Rosario, Embu, São Paulo Brazil, in Terra 93: 7a Conferência Internacional sobre o Estudo e Conservação da Arquitectura de Terra. Direcção Geral dos Edifícios e Monumentos Nacionais, Lisbon.

Rainer L. (2018). Deterioration and Pathology of Earthen Architecture, in Avrami, E., Guillaud, G., Hardy, M. (Eds). Terra Literature Review. An Overview of Research in Earthen Architecture Conservation. Getty Conservation Institute, Los Ángeles, 45-61.

Tomasi, J. (2012). Mirando lo vernáculo. Tradiciones disciplinares en el estudio de "otras arquitecturas" en la Argentina del siglo XX. Revista Área, 17, 68-83.

Tomasi, J. (2013). Cubiertas con tierra en el área puneña. Acercamiento a las técnicas y prácticas contemporáneas en Susques (Jujuy, Argentina), in $13^{\circ}$ SIACOT. Valparaíso, Chile.

Tomasi, J., Barada, J. (2020). Recurring damages on earthen heritage. Diagnosis and possible interventions in the highlands of Jujuy (Argentina). Journal of Building Pathology and Rehabilitation, 5, 28.

Viñuales, G. (2011). Monumentos Nacionales de adobe y otras técnicas de tierra. Boletín Informativo de la Comisión Nacional de Monumentos, Buenos Aires, 21-29.

Viscontini, E. (1968). Humahuaca y su templo. Dirección Provincial de Cultura, San Salvador de Jujuy. 